\title{
Association between dry eye and depressive symptoms in an elderly Chinese population in Taiwan: the Shihpai Eye Study
}

\author{
Tung-Mei Kuang ${ }^{1,2,3} \cdot$ Su-Ying Tsai ${ }^{4} \cdot$ Catherine Jiu-Ling Liu ${ }^{1,2} \cdot$ Shui-Mei Lee ${ }^{1,2} \cdot$ Pesus Chou $^{3}$
}

Received: 28 March 2020 / Accepted: 13 November 2020 / Published online: 30 November 2020

(c) The Author(s), under exclusive licence to The Royal College of Ophthalmologists 2020

\begin{abstract}
Background Dry eye may impact quality of life and daily activities and depression is a widespread illness. Many studies showed the two diseases often coexist. However, studies were limited to retrospective chart review. This study aimed to investigate the association between dry eye and depressive symptoms in an older Asian population.

Methods The Shihpai Eye Study was a community-based, cross-sectional survey of vision and eye diseases among noninstitutionalized subjects 65 years of age and older in Shihpai, Taipei, Taiwan. Residents fulfilling these criteria were randomly selected to be invited to participate in the study, which included a questionnaire and an eye examination conducted between July 1, 1999 and December 31, 2000.

Results Of the 2045 subjects recruited, $1361(66.6 \%)$ completed the examination. 8.8\% (95\% confidence interval (CI): 7.3-10.3\%) of the participants were diagnosed to have depressive symptoms. Under multivariate analysis, depressive symptoms were significantly associated with frequent symptoms of dry eye (odds ratio (OR): 1.97, 95\% CI: 1.36-2.92; $p<$ 0.001 ). None of the dry eye signs was associated with depressive symptoms. For participants reporting frequent symptoms, tear-film break-up time $\leq 10 \mathrm{~s}$ (OR: $2.06,95 \%$ CI: 1.38-3.05; $p<0.001)$, Schirmer test score $\leq 5 \mathrm{~mm}$ (OR: $2.01,95 \%$ CI: $1.33-3.03 ; p<0.001$ ), and meibomian gland disease (OR: $1.99,95 \%$ CI: $1.31-3.01 ; p=0.001$ ) were significantly related to depressive symptoms. Fluorescein staining of the cornea was not correlated to depressive symptoms in participants with dry eye symptoms.
\end{abstract}

Conclusions Depressive symptoms are more highly correlated with dry eye symptoms than dry eye signs.

\section{Introduction}

Dry eye is a common ocular disease leading to discomfort and visual disturbances. It is a multifactorial disorder of the tears and ocular surface that causes tear-film instability, and may impair the quality of life and daily activities [1-4]. Despite the availability of clinical examinations to evaluate

Tung-Mei Kuang

kuangtammy@gmail.com

1 Department of Ophthalmology, Taipei Veterans General Hospital, Taipei, Taiwan

2 National Yang-Ming University, School of Medicine, Taipei, Taiwan

3 Community Medicine Research Center and Institute of Public Health, National Yang-Ming University, Taipei, Taiwan

4 Department of Health Management, I-Shou University, Kaohsiung, Taiwan different parameters of the ocular surface, symptoms often do not correlate well with the test results $[5,6]$. Discrepancies between the ocular symptoms and signs of dry eye are common. Interestingly, the symptoms of dry eye are more highly correlated with non-ocular conditions than with dry eye signs [7].

Depression is a widespread illness, affecting more than 300 million people worldwide. According to the World Health Organization, depression was an important cause of the global disease burden in 2004 and will be the leading cause by 2030 .

Dry eye and depressive symptoms often coexist. Depression scores are highly correlated with dry eye symptoms, but only a few dry eye signs [8]. In addition, both dry eye symptoms and dry eye signs are more prevalent in depressive and anxious study participants [9]. Previous studies linking depression, anxiety, and dry eye signs and symptoms were mostly limited to retrospective chart reviews $[3,4,10]$ and few prospective interventional case series [11] have confirmed that effective treatment of 
dry eye disease may positively affect depressive symptoms and anxiety. Two studies [8, 12] assessed this issue in a population-based design and only one of these targeted an older population [8]. The present study investigated the association between dry eye and depressive symptoms in an older Asian population.

\section{Methods}

The Shihpai Eye Study [13-15] was a community-based, cross-sectional survey of vision and eye diseases among noninstitutionalized subjects 65 years of age and older in Shihpai, Taipei, Taiwan. We planned to recruit a random sample of $\sim 2000$ residents 65 years of age or older with complete baseline information who were identified from the household registration system. This system officially registers personal information such as date of birth, sex, and home address, as well as family members and relations. According to the official household registration figures for 1999 , the total number of residents 65 years of age or older in Shihpai was 4750. A total of 3746 residents were eligible for the study after excluding vacant households (658 persons); residents who died before they could be contacted (48 persons); and inpatient, paralyzed, and disabled residents (298 persons). Of these 3746 eligible subjects, 2045 were randomly selected and invited to participate in the survey [14]. The baseline examination was conducted between July 1, 1999 and December 31, 2000. A questionnaire was used to obtain information on demographics (age, sex, locality, marital status, and education), dry eye, and depressive symptoms. Personal medical history was assessed by a checklist. The participants were asked whether a physician had diagnosed them with a chronic disease, such as diabetes (yes/no), hypertension (yes/no), cardiovascular disease (yes/no), or stroke (yes/no). Subjects that were interviewed were invited to participate in a comprehensive ophthalmic examination conducted at the Taipei Veterans General Hospital. Ophthalmologists conducted the examinations according to a standardized protocol. After receiving an explanation of the purpose and procedure of the study, each subject provided written informed consent to participate in the study. The survey followed the tenets of the Declaration of Helsinki. This study was approved by the Institutional Review Board of the Taipei Veterans General Hospital (VGHIRB 89-11-01A).

\section{Dry eye questionnaire}

The dry eye symptom questionnaire included eight symptoms (Table 1). Six of the items were the same as those used by Schein et al. [16], we added two more symptomsstickiness (question 4) and tearing (question 5)—because
Table 1 Dry eye questionnaire ${ }^{\mathrm{a}}$.

1. Do your eyes ever feel dry?

2. Do you ever feel a gritty or sandy sensation in your eyes?

3. Do your eyes ever have a burning sensation?

4. Do your eyes ever feel sticky?

5 . Do your eyes ever feel watery or tearing?

6. Are your eyes ever red?

7. Do you notice much crusting or discharge on your lashes?

8. Do your eyes ever get stuck shut in the morning?

aAllowable responses: never, rarely, sometimes, often, or all of the time.

these are very common complaints of dry eye patients and have also been included in the questionnaires of other dry eye studies. When a respondent indicated the presence of a symptom, he or she was asked to indicate whether the symptom was experienced rarely, sometimes, often, or all of the time.

\section{Dry eye examination}

Instillation of anesthetics or manipulation of the lid may affect the tear-film break-up time; therefore, this test was performed before the other dry eye tests. To determine the tear-film break-up time, subjects were asked to look upward, and a dry fluorescein strip lightly touched the inferior palpebral conjunctiva as the lower lid was retracted gently. The subject was asked to blink several times and then hold the eye open. The tear film was observed with a slit lamp and a cobalt-blue filter. The time it took for the tear film to break, as indicated by the appearance of a black spot or line, was recorded as the tear-film break-up time. The test was repeated three times for each eye, and the average tearfilm break-up time was recorded.

A thorough slit-lamp examination of the eyelid margin, conjunctiva, cornea, and anterior segment was then performed. Fluorescein staining of the cornea was graded as 0 (no staining), 1 (mild staining with a few disseminated stains and limited to less than one-third of the cornea), 2 (moderate staining with severity between grades 1 and 3), or 3 (severe staining with confluent stains and occupying half or more of the cornea). The condition of the meibomian glands was determined by observing the lid margin with a slit lamp. The presence of lid margin telangiectasia and grading of meibomian gland obstruction (grade 0 , no obstruction; grade 1, plugging with translucent serous secretion when compressing the lid margin; grade 2, plugging with viscous or waxy white secretion when compressing the lid margin; and grade 3 , plugging with no secretion when compressing the lid margin) were recorded.

The Schirmer test was performed after the slit-lamp examination, so that ocular irritation by the test strip would 
not interfere with other examination results. One minute after instilling a drop of $0.5 \%$ proparacaine, a cotton swab was used to gently dry any visible fluid in the inferior fornix and lid margin. A precalibrated filter strip (Color Bar; EagleVision, Inc., Memphis, TN) was then placed temporally in each lower fornix and left in place for $5 \mathrm{~min}$. The patient was allowed to either blink normally or to close his or her eyes. After $5 \mathrm{~min}$, the strips were removed, and the amount of wetting (in millimeters) was recorded from the precalibrated strips.

\section{Definition}

Dry eye was defined as the presence of one or more symptoms often or all the time. Positive signs were defined as one or both eyes with a tear-film break-up time of $\leq 10 \mathrm{~s}$, a Schirmer test score of $\leq 5 \mathrm{~mm}$, fluorescein score of $\geq 1$, or the existence of meibomian gland disease. Meibomian gland disease was defined as telangiectasia at the lid margin or plugging of the gland orifices (grade $\geq 1$ ). A cutoff of $10 \mathrm{~s}$ was chosen for the tear-film break-up time and a cutoff of $5 \mathrm{~mm}$ was selected for the Schirmer score because these two values were used in previous studies and are commonly adopted in clinical practice.

\section{Geriatric Depression Scale-Short Form (GDS-S)}

A Chinese version of the GDS-S [17] was used to screen for depression. The Geriatric Depression Scale (GDS) has been successfully translated into many languages (Chinese, Dutch, French, German, Hebrew, Italian, Japanese, Portuguese, Rumanian, Russian, Spanish, and Yiddish), is used in several countries around the world, and has been extensively validated in older populations and settings [18, 19]. The GDS is a commonly used instrument consisting of 30 items with yes/no answers [20]. The short form, or GDS-S (Table 2), contains 15 of the items and was developed to reduce fatigue and deteriorating concentration in older individuals [21]. Scores on the GDS-S are a highly correlated with those on the original form, and scores of 5 or more on the GDS-S are associated with a clinical diagnosis of depression [22, 23]. A translated and adapted Chinese-language version of the GDS has been developed, and the GDS-S of this Chineselanguage version correctly classified slightly over $90 \%$ of cases and non-cases in a community survey [17]. In view of these results, we used the Chinese-language version of the GDS-S instrument, which was extensively documented as reliable and valid in past studies to explore the association between visual impairment and depression. In fact, the internal consistency of reliability of the Chineselanguage version of the GDS-S used in our study was high (Cronbach's alpha: 0.80-0.83).
Table 2 Geriatric Depression Scale-Short Form.

1. Are you basically satisfied with your life?

2. Have you dropped many of your activities and interests?

3. Do you feel that your life is empty?

4. Do you often get bored?

5. Are you in good spirits most of the time?

6. Are you afraid that something bad is going to happen to you?

7. Do you feel happy most of the time?

8. Do you often feel helpless?

9. Do you prefer to stay at home, rather than going out and doing new things?

10. Do you feel you have more problems with memory than most?

11. Do you think it is wonderful to be alive now?

12. Do you feel pretty worthless the way you are now?

13. Do you feel full of energy?

14. Do you feel that your situation is hopeless?

15. Do you think that most people are better off than you are?

\section{Statistical analysis}

Chi-square analysis was used to evaluate whether each independent variable (symptoms and signs of dry eye) was significantly associated with depressive symptoms (dependent variable). A $p$ value of $<0.05$ was considered to be statistically significant. Multivariate logistic regression analysis was used with depressive symptoms as the dependent variable and symptoms and signs of dry eye as independent variables. Sex, education level, diabetes mellitus, and stroke were also adjusted in each logistic regression. The analysis was performed using Statistical Analysis System (SAS 6.12; SAS Institute, Cary, NC) software.

\section{Results}

Of the 2045 subjects randomly recruited, $2038(99.7 \%)$ cooperated with the household interview and completed the questionnaire; 7 subjects $(0.3 \%)$ could not be contacted after three attempts and were not interviewed. After completing the questionnaire, 1361 subjects $(66.6 \%)$ completed the eye examinations. The remaining 677 subjects $(33.1 \%)$ did not undergo the eye examination.

A comparison of demographic information and some of the variables in subjects who did and did not undergo the eye examination revealed that the participating subjects were younger than those who refused to participate in the study ( 72.2 vs. 74.3 years, $t=5.10, P<0.001)$, were more likely to be male $\left(\chi^{2}=24.8, p<0.001\right)$, and were more educated $\left(\chi^{2}=14.16, \quad p<0.001\right)$. The other variables (marital status and history of diabetes and hypertension) did not differ significantly between the two groups [24]. The 
Table 3 Demographics characteristics of study subjects.

\begin{tabular}{|c|c|c|c|c|}
\hline \multirow[b]{2}{*}{ Variable } & \multirow[b]{2}{*}{ Total $(\%)$} & \multicolumn{3}{|c|}{ Depression (GDS-S) } \\
\hline & & Yes $(\%)$ & No $(\%)$ & $p$ value \\
\hline GDS-S $\geq 5$ & & 8.8 & 91.2 & - \\
\hline $\begin{array}{l}\text { Age, years } \\
\text { (means, range) }\end{array}$ & $\begin{array}{l}72.2 \\
(65-91.4)\end{array}$ & & & \\
\hline$\geq 75$ years & 28.5 & 37.8 & 27.6 & 0.0191 \\
\hline $65-74$ years & 71.5 & 62.2 & 72.3 & \\
\hline \multicolumn{5}{|l|}{ Gender } \\
\hline Male & 60.3 & 40.3 & 62.2 & $<0.0001$ \\
\hline Female & 39.7 & 59.7 & 37.8 & \\
\hline \multicolumn{5}{|l|}{ Level of education } \\
\hline $\begin{array}{l}\text { Primary school and } \\
\text { illiterate }\end{array}$ & 47.4 & 63.9 & 45.8 & 0.0002 \\
\hline $\begin{array}{l}\text { Junior high school } \\
\text { and above }\end{array}$ & 52.6 & 36.1 & 54.2 & \\
\hline $\begin{array}{l}\text { Has current } \\
\text { smoking habit }\end{array}$ & 17.8 & 18.5 & 17.7 & 0.8259 \\
\hline Has hypertension & 45.0 & 52.1 & 44.3 & 0.1016 \\
\hline Has diabetes mellitus & 25.2 & 36.1 & 24.2 & 0.0041 \\
\hline Has stroke & 4.8 & 10.9 & 4.2 & 0.0011 \\
\hline
\end{tabular}

mean age of the participants was 72.2 years (range, 65-91 years), and $60.4 \%$ were men.

Among the 1361 participants, 8.8\% (95\% confidence interval (CI): $7.3-10.3 \%$ ) was with depressive symptoms. The very old ( $\geq 75$ years old; $p<0.05$ ), female participants $(p<0.0001)$, and participants with lower educational level $(p<0.001)$ were more likely to have depressive symptoms. Also, subjects with a history of diabetes mellitus $(p<0.05)$ and stroke $(p=0.001)$ were more likely to have depressive symptoms (Table 3 ).

Table 4 shows the prevalence of symptoms and signs of dry eye according to sex. Females were more likely to report frequent symptoms of dry eye and had a Schirmer test score $\leq 5 \mathrm{~mm}$. On the other hand, female participants with frequent symptoms were more likely to have a tearfilm break-up time $\leq 10 \mathrm{~s}$, a Schirmer test score $\leq 5 \mathrm{~mm}$, and a fluorescein stain score of at least 1 . On the other hand, subjects having frequent dry eye symptoms were more likely to have symptoms of depression. No dry eye sign alone was associated with depression. Participants reporting one or more symptom often or all of the time and having a tear-film break-up time $\leq 10 \mathrm{~s}$, a Schirmer test score $\leq 5 \mathrm{~mm}$, and meibomian gland disease were more likely to have depressive symptoms. Table 5 shows the association between symptoms and signs of dry eye and depressive symptoms.

Under univariate analysis, frequent dry eye symptoms (odds ratio (OR): $2.31,95 \%$ CI: $1.58-3.38 ; p<0.001$ ) were significantly associated with depressive symptoms. None of the dry eye signs alone was related to depressive symptoms.
On the other hand, frequent dry eye symptoms and having positive signs of a tear-film break-up time $\leq 10 \mathrm{~s}$ (OR: 2.35, 95\% CI: $1.59-3.43 ; p<0.001)$, Schirmer test score $\leq 5 \mathrm{~mm}$ (OR: $2.32,95 \%$ CI: $1.56-3.47 ; p<0.001$ ), and meibomian gland disease (OR: $2.25,95 \%$ CI: $1.51-3.37 ; p<0.001$ ) were positively associated with depressive symptoms. The fluorescein stain score was the only sign not associated with depressive symptoms despite the presence of dry eye symptoms.

Under multivariate analysis adjusting for sex, educational level, diabetes mellitus, and stroke, the results were similar. Depression was significantly associated with frequent symptoms of dry eye (OR: 1.97, 95\% CI: 1.36-2.92; $p<$ 0.001 ). None of the dry eye signs alone was associated with depressive symptoms. For participants with frequent dry eye symptoms, a tear-film break-up time $\leq 10 \mathrm{~s}$ (OR: $2.06,95 \%$ CI: $1.38-3.05 ; p<0.001$ ), a Schirmer test score $\leq 5 \mathrm{~mm}$ (OR: 2.01, 95\% CI: $1.33-3.03 ; p<0.001)$, and meibomian gland disease (OR: $1.99,95 \%$ CI: $1.31-3.01 ; p=0.001$ ) were significantly related to depressive symptoms. The fluorescein stain score was not related to depressive symptoms in participants with dry eye symptoms.

\section{Discussion}

After World Mental Health Day was initiated by the World Federation for Mental Health in 1992, many countries adopted it as a means of promoting mental health. Depression was selected as the main theme in 2012. Depression can affect anyone and is one of the most widespread illnesses in the world. Moreover, depression often coexists with other serious diseases such as stroke [22], trauma [4], and dry eye [3, 12, 25]. Dry eye disease may adversely affect daily life [1-4] and is common among the older persons [26]. It is also one of the most frequently encountered conditions in ophthalmic clinics.

A recent meta-analysis revealed that dry eye was associated with a threefold prevalence of depression [25]. The study included mostly retrospective chart reviews. A prospective interventional study [11] demonstrated that dry eye can be effectively treated in most cases and that treating dry eye may alleviate the patient's psychological burden. Our findings indicate that an older population with dry eye disease was nearly two times more likely to have depressive symptoms. This finding is in accordance with an OR of 2.5 in a survey conducted among community-dwelling older persons in Korea [8]. The mechanism and causal relationship between dry eye and depressive symptoms is not clear. Dry eye symptoms negatively affect the quality of life, performance of daily activities, emotional well-being, and working capacity [27]. The chronic discomfort and pain from dry eye might also influence cognitive processes, 
Table 4 Prevalence of symptoms and signs of dry eye according to gender and depression in Shihpai.

\begin{tabular}{|c|c|c|c|c|c|c|c|c|}
\hline \multirow[b]{2}{*}{ Variable } & \multicolumn{2}{|c|}{ Total $(n=1361)$} & \multicolumn{3}{|l|}{ Gender } & \multicolumn{3}{|c|}{ Depression (GDS-S) } \\
\hline & $n$ & $\%$ & Male $(\%)$ & Female $(\%)$ & $\begin{array}{l}\text { Gender difference } \\
\left(\chi^{2} \text { test }\right)\end{array}$ & Yes $(\%)$ & No $(\%)$ & $\begin{array}{l}\text { Group difference } \\
\left(\chi^{2} \text { test }\right)\end{array}$ \\
\hline \multicolumn{9}{|c|}{ Symptoms and signs of dry eye } \\
\hline Frequent symptoms ${ }^{\mathrm{a}}$ & 459 & 33.7 & 30.2 & 39.2 & 0.001 & 52.1 & 32.0 & $<0.0001$ \\
\hline $\mathrm{BUT} \leq 10 \mathrm{~s}$ & 1079 & 79.3 & 78.8 & 80.0 & 0.615 & 84.0 & 78.8 & 0.1750 \\
\hline $\mathrm{F} / \mathrm{S} \geq 1^{\mathrm{b}}$ & 454 & 33.4 & 31.6 & 36.0 & 0.095 & 31.9 & 33.6 & 0.7166 \\
\hline Schirmer test $\leq 5 \mathrm{~mm}$ & 795 & 58.4 & 55.4 & 63.1 & 0.005 & 66.4 & 57.7 & 0.0652 \\
\hline $\mathrm{MGD}^{\mathrm{c}}$ & 828 & 60.8 & 62.8 & 57.9 & 0.071 & 66.4 & 60.5 & 0.2090 \\
\hline \multicolumn{9}{|c|}{ Both reporting one or more symptoms often or all time and having positive signs } \\
\hline $\mathrm{BUT} \leq 10 \mathrm{~s}$ & 362 & 26.6 & 24.0 & 30.6 & 0.007 & 43.7 & 24.9 & $<0.0001$ \\
\hline $\mathrm{F} / \mathrm{S} \geq 1^{\mathrm{b}}$ & 154 & 11.3 & 9.6 & 13.9 & 0.014 & 15.1 & 11.0 & 0.1792 \\
\hline Schirmer test $\leq 5 \mathrm{~mm}$ & 287 & 21.1 & 17.8 & 26.2 & 0.001 & 36.1 & 19.6 & $<0.0001$ \\
\hline $\mathrm{MGD}^{\mathrm{c}}$ & 283 & 20.8 & 19.2 & 23.2 & 0.078 & 35.3 & 19.5 & $<0.0001$ \\
\hline
\end{tabular}

$B U T$ tear break-up time, $F / S$ fluorescein stain score of the cornea, $M G D$ meibomian gland disease.

${ }^{a}$ Defined as reporting one or more symptoms often or all of the time.

${ }^{\mathrm{b}} 0$, none; 1 , mild; 2 , moderate; 3 , severe.

${ }^{c}$ Positive anatomic abnormalities of the meibomian gland, such as plugging and lid margin telangiectasia.

Table 5 Univariate and multivariate analyses of the relation between symptoms and signs of dry eye and depression among residents 65 years of age and older in Shihpai, Taipei, Taiwan $(n=1352)$.

\begin{tabular}{|c|c|c|c|c|c|c|}
\hline \multirow[b]{3}{*}{ Variable } & \multicolumn{6}{|c|}{ Depressive symptoms $(\text { GDS-S })^{\mathrm{a}}$} \\
\hline & \multicolumn{3}{|c|}{ Univariate $\left(\chi^{2}\right.$ test $)$} & \multicolumn{3}{|c|}{ Multivariate $^{\mathrm{e}}$ (logistic regressions) } \\
\hline & OR & $95 \% \mathrm{CI}$ & $p$ & OR & $95 \% \mathrm{CI}$ & $p$ \\
\hline \multicolumn{7}{|c|}{ Symptoms and signs of dry eye } \\
\hline Frequent symptoms ${ }^{\mathrm{b}}$ & 2.31 & $1.58-3.38$ & $<0.0001$ & 1.97 & $1.36-2.92$ & 0.0006 \\
\hline $\mathrm{BUT} \leq 10 \mathrm{~s}$ & 1.42 & $0.85-2.36$ & 0.1750 & 1.42 & $0.86-2.45$ & 0.1850 \\
\hline $\mathrm{F} / \mathrm{S} \geq 1^{\mathrm{c}}$ & 0.92 & $0.62-1.38$ & 0.7166 & 0.86 & $0.56-1.29$ & 0.4802 \\
\hline Schirmer test $\leq 5 \mathrm{~mm}$ & 1.45 & $0.97-2.15$ & 0.0652 & 1.36 & $0.91-2.06$ & 0.1298 \\
\hline $\mathrm{MGD}^{\mathrm{d}}$ & 1.28 & $0.86-1.91$ & 0.2088 & 1.29 & $0.86-1.96$ & 0.2100 \\
\hline \multicolumn{7}{|c|}{ Both reporting one or more symptoms often or all time and having positive signs } \\
\hline $\mathrm{BUT} \leq 10 \mathrm{~s}$ & 2.35 & $1.59-3.43$ & $<0.0001$ & 2.06 & $1.38-3.05$ & 0.0003 \\
\hline $\mathrm{F} / \mathrm{S} \geq 1^{\mathrm{c}}$ & 1.43 & $0.84-2.44$ & 0.1792 & 1.14 & $0.63-1.94$ & 0.6341 \\
\hline Schirmer test $\leq 5 \mathrm{~mm}$ & 2.32 & $1.56-3.47$ & $<0.0001$ & 2.01 & $1.33-3.03$ & 0.0008 \\
\hline $\mathrm{MGD}^{\mathrm{d}}$ & 2.25 & $1.51-3.37$ & $<0.0001$ & 1.99 & $1.31-3.01$ & 0.0010 \\
\hline
\end{tabular}

$B U T$ tear break-up time, $O R$ odds ratio, $C I$ confidence interval, $F / S$ fluorescein stain score of the cornea, $M G D$ meibomian gland disease.

${ }^{\mathrm{a}} G D S-S$ Geriatric Depression Scale-Short Form.

${ }^{b}$ Defined as reporting one or more symptoms often or all of the time.

${ }^{\mathrm{c}} 0$, none; 1 , mild; 2 , moderate; 3 , severe.

${ }^{\mathrm{d} P o s i t i v e ~ a n a t o m i c ~ a b n o r m a l i t i e s ~ o f ~ t h e ~ m e i b o m i a n ~ g l a n d, ~ s u c h ~ a s ~ p l u g g i n g ~ a n d ~ l i d ~ m a r g i n ~ t e l a n g i e c t a s i a . ~}$

${ }^{\mathrm{e}}$ Multivariate logistic regressions adjusting for gender, education level, diabetes mellitus, and stroke.

sleep, mood, and mental health [27, 28]. On the other hand, somatization is common in depression [29]. Depressive subjects have a lower threshold for perceiving physical discomfort and pain [30]. In addition, medications used to treat depression such as antidepressants with anticholinergic effects [31], selective serotonin reuptake inhibitors, and serotonin-norepinephrine reuptake inhibitors may further increase the risk of dry eye [32]. Because the current literature is mostly based on retrospective chart reviews and cross-sectional studies, additional studies are required to evaluate the causal relationship between dry eye and depressive symptoms. 
Our results provided further evidence that discrepancies exist between dry eye signs and symptoms. Consistent with the literature, we noted that depressive symptoms are more highly correlated with dry eye symptoms than dry eye signs $[4,7]$. After adjusting for other variables, none of the dry eye signs alone was associated with depressive symptoms. This is in agreement with other studies finding that objective dry eye parameters are not associated with the depression score $[8,12]$. Our findings conflict with those of Kim et al., however, in the aspect that the association between dry eye symptoms and depression was significant in our older population irrespective of the Schirmer test score as opposed to only those with a Schirmer test score $\geq$ 5. Only when combined with dry eye symptoms were tearfilm break-up time, the Schirmer test score, and meibomian gland disease significantly related to depressive symptoms. It is not clear why disruption of the corneal epithelium with fluorescein score $\geq 1$ is not correlated with depressive symptoms even in those with dry eye symptoms, and this finding warrants further investigation. It is logical that the subjective sensitivity and discomfort experienced by those with dry eye disease would affect their mood and despondency. The visual disturbance caused by dry eye signs may further aggravate a depressive condition.

Interrelation of the two conditions is biologically plausible. Female participants are more prone to both conditions, which may be due to sex hormones. The clinical severity of dry eye is correlated with interleukin (IL)-17 and tumor necrosis factor- $\alpha$ levels. Levels of these two types of inflammatory cytokines are also higher in the tear fluid of depressive individuals [33]. A higher omega6-omega-3 ratio promotes the production of IL-1, IL-6, and tumor necrosis factor- $\alpha$. On the other hand, the antiinflammatory potential of omega-3 polyunsaturated fatty acids alleviates both dry eye signs and symptoms [34] as well as depression [30]. This implies that these two diseases may have common pathologic mechanisms. Further clarification of the interplay between different proinflammatory cytokines in the pathogenesis of ocular findings in depressive patients may be helpful in the treatment of these patients.

Our study has some limitations. Potential subjects who were institutionalized were not examined. Excluding inpatients, the paralyzed and disabled may have accounted for a disproportionate number of people with depressive symptoms and removing them from the subject pool may have biased our results. Hence, the association between depression and dry eye might be greater than our results indicate. On the other hand, our questionnaire did not focus on a specific drug history especially antidepressants of older persons. Furthermore, our participants were more likely to be younger, higher educated, and male, and our results indicate that older persons, females, and the less educated are more likely to have depressive symptoms. Hence, the extent and effects of depressive symptoms should be even greater than our results revealed. Despite these limitations, this is one of the few community-based studies of dry eye and depressive symptoms among older persons in Asia. The response rate in our study was relatively low (66.6\% of those eligible). Obtaining populationbased prevalence estimates of eye disease among older persons is challenging because this group of individuals is less likely to participate in research studies [35]. The inclusion rate in the Rotterdam Study ranged from $59 \%$ in the group aged $75-84$ years to $28 \%$ in the group aged 85 years and older. Similarly, in the Baltimore Study [36] inclusion rates were $48 \%$ in the group aged 70-79 years and $21 \%$ in the group aged 80 years and older. Another potential reason for the low participation rate is that the lack of utilization of ophthalmic care for prevention and treatment has created the impression that loss of vision is expected in senior life and that nothing can be done to improve the situation among older persons, particularly those less educated [13]. Many older persons were not aware of the importance of regular physical checkups or that many diseases are asymptomatic in the early stages. This possibility is supported by the findings that more than $90 \%$ of participants had received previous contact eye service, whereas only $8 \%$ of the nonparticipants had previously received similar services [15]. We further confirmed this speculation by asking the nonparticipants about their reasons for declining the ophthalmic examination: $63 \%$ felt that they were fine and did not need any examination; $10 \%$ of the older persons stated that they already had regular ophthalmic clinical follow-ups; and some responded that they had previously participated in a similar survey [15].

Ophthalmologists should be aware that older persons with dry eye signs and symptoms may have comorbidities like anxiety and depression. It would be optimal for older persons with dry eye symptoms to be screened for depressive symptoms. Referral of suspected cases to psychiatry for diagnosis and treatment is appropriate.

\section{Summary}

\section{What was known before}

- Dry eye is associated with depression retrospective chart review Caucasian population.

\section{What this study adds}

- Dry eye symptoms are associated with depressive symptoms in an elderly Chinese population. 
- Dry eye signs, on the other hand, are correlated with depressive symptoms, only in participants with frequent dry eye symptoms.

Acknowledgements This study was supported by a grant from the Taipei Veterans General Hospital (VGH89-404-1, VGH90-445-1, and VGH91-382-1) and awarded by the Medical Research and Advancement Foundation in Memory of Dr. Chi-Shuen Tsou.

\section{Compliance with ethical standards}

Conflict of interest The authors declare that they have no conflict of interest.

Publisher's note Springer Nature remains neutral with regard to jurisdictional claims in published maps and institutional affiliations.

\section{References}

1. Li M, Gong L, Chapin WJ, Zhu M. Assessment of vision-related quality of life in dry eye patients. Invest Ophthalmol Vis Sci. 2012;53:5722-7.

2. Mertzanis P, Abetz L, Rajagopalan K, Espindle D, Chalmers R, Snyder C, et al. The relative burden of dry eye in patients' lives: comparisons to a U.S. normative sample. Invest Ophthalmol Vis Sci. 2005;46:46-50.

3. van der Vaart R, Weaver MA, Lefebvre C, Davis RM. The association between dry eye disease and depression and anxiety in a large population-based study. Am J Ophthalmol. 2015;159: 470-4.

4. Galor A, Feuer W, Lee DJ, Florez H, Faler AL, Zann KL, et al. Depression, post-traumatic stress disorder, and dry eye syndrome: a study utilizing the national United States Veterans Affairs administrative database. Am J Ophthalmol. 2012;154:340-6.e2.

5. Schein OD, Tielsch JM, Munoz B, Bandeen-Roche K, West S. Relation between signs and symptoms of dry eye in the elderly. A population-based perspective. Ophthalmology. 1997; 104:1395-401.

6. Nichols KK, Mitchell GL, Zadnik K. The repeatability of clinical measurements of dry eye. Cornea. 2004;23:272-85.

7. Galor A, Felix ER, Feuer W, Shalabi N, Martin ER, Margolis TP, et al. Dry eye symptoms align more closely to non-ocular conditions than to tear film parameters. Br J Ophthalmol. 2015;99: 1126-9.

8. Kim KW, Han SB, Han ER, Woo SJ, Lee JJ, Yoon JC, et al. Association between depression and dry eye disease in an elderly population. Invest Ophthalmol Vis Sci. 2011;52:7954-8.

9. Wen W, Wu Y, Chen Y, Gong L, Li M, Chen X, et al. Dry eye disease in patients with depressive and anxiety disorders in Shanghai. Cornea. 2012;31:686-92.

10. Segal BM, Pogatchnik B, Holker E, Liu H, Sloan J, Rhodus N, et al. Primary Sjogren's syndrome: cognitive symptoms, mood, and cognitive performance. Acta Neurol Scand. 2012;125:272-8.

11. Bitar MS, Olson DJ, Li M, Davis RM. The correlation between dry eyes, anxiety and depression: the Sicca, Anxiety and Depression Study. Cornea. 2019;38:684-9.

12. Labbe A, Wang YX, Jie Y, Baudouin C, Jonas JB, Xu L. Dry eye disease, dry eye symptoms and depression: the Beijing Eye Study. Br J Ophthalmol. 2013;97:1399-403.
13. Tsai SY, Hsu WM, Cheng CY, Liu JH, Chou P. Epidemiologic study of age-related cataracts among an elderly Chinese population in Shih-Pai, Taiwan. Ophthalmology. 2003;110:1089-95.

14. Cheng CY, Hsu WM, Liu JH, Tsai SY, Chou P. Refractive errors in an elderly Chinese population in Taiwan: the Shihpai Eye Study. Invest Ophthalmol Vis Sci. 2003;44:4630-8.

15. Kuang TM, Tsai SY, Liu CJ, Ko YC, Lee SM, Chou P. Sevenyear incidence of uncorrected refractive error among an elderly Chinese population in Shihpai, Taiwan: the Shihpai Eye Study. Eye. 2016;30:570-6.

16. Schein OD, Munoz B, Tielsch JM, Bandeen-Roche K, West S. Prevalence of dry eye among the elderly. Am J Ophthalmol. 1997; 124:723-8.

17. Chiu HF, Lee HC, Wing YK, Kwong PK, Leung CM, Chung DW. Reliability, validity and structure of the Chinese Geriatric Depression Scale in a Hong Kong context: a preliminary report. Singapore Med J. 1994;35:477-80.

18. Sheikh JI, Yesavage JA, Brooks JO 3rd, Friedman L, Gratzinger $\mathrm{P}$, Hill RD, et al. Proposed factor structure of the Geriatric Depression Scale. Int Psychogeriatr. 1991;3:23-8.

19. Montorio I, Izal M. The Geriatric Depression Scale: a review of its development and utility. Int Psychogeriatr. 1996;8:103-12.

20. Yesavage JA, Brink TL, Rose TL, Lum O, Huang V, Adey M, et al. Development and validation of a geriatric depression screening scale: a preliminary report. J Psychiatr Res. 1982;17: $37-49$.

21. Burke WJ, Roccaforte WH, Wengel SP. The short form of the Geriatric Depression Scale: a comparison with the 30-item form. J Geriatr Psychiatry Neurol. 1991;4:173-8.

22. Fuh JL, Liu HC, Wang SJ, Liu CY, Wang PN. Poststroke depression among the Chinese elderly in a rural community. Stroke. 1997;28:1126-9.

23. Alden D, Austin C, Sturgeon R. A correlation between the Geriatric Depression Scale long and short forms. J Gerontol. 1989;44: P124-5.

24. Kuang TM, Tsai SY, Hsu WM, Cheng CY, Liu JH, Chou P. Body mass index and age-related cataract: the Shihpai Eye Study. Arch Ophthalmol. 2005;123:1109-14.

25. Wan KH, Chen LJ, Young AL. Depression and anxiety in dry eye disease: a systematic review and meta-analysis. Eye. 2016;30: 1558-67.

26. Lin PY, Tsai SY, Cheng CY, Liu JH, Chou P, Hsu WM. Prevalence of dry eye among an elderly Chinese population in Taiwan: the Shihpai Eye Study. Ophthalmology. 2003;110:1096-101.

27. Pouyeh B, Viteri E, Feuer W, Lee DJ, Florez H, Fabian JA, et al. Impact of ocular surface symptoms on quality of life in a United States veterans affairs population. Am J Ophthalmol. 2012;153: 1061-6.e3.

28. Fine PG. Long-term consequences of chronic pain: mounting evidence for pain as a neurological disease and parallels with other chronic disease states. Pain Med. 2011;12:996-1004.

29. Katon W, Kleinman A, Rosen G. Depression and somatization: a review. Part I Am J Med. 1982;72:127-35.

30. Kiecolt-Glaser JK, Belury MA, Porter K, Beversdorf DQ, Lemeshow S, Glaser R. Depressive symptoms, omega-6:omega-3 fatty acids, and inflammation in older adults. Psychosom Med. 2007;69:217-24.

31. Wong J, Lan W, Ong LM, Tong L. Non-hormonal systemic medications and dry eye. Ocul Surf. 2011;9:212-26.

32. Kocer E, Kocer A, Ozsutcu M, Dursun AE, Krpnar I. Dry eye related to commonly used new antidepressants. J Clin Psychopharmacol. 2015;35:411-3.

33. Mrugacz M, Ostrowska L, Bryl A, Szulc A, Zelazowska-Rutkowska B, Mrugacz G. Pro-inflammatory cytokines associated with clinical severity of dry eye disease of patients with depression. Adv Med Sci. 2017;62:338-44. 
34. Miljanovic B, Trivedi KA, Dana MR, Gilbard JP, Buring JE, Schaumberg DA. Relation between dietary n-3 and n-6 fatty acids and clinically diagnosed dry eye syndrome in women. Am J Clin Nutr. 2005;82:887-93.

35. Friedman DS, Jampel HD, Munoz B, West SK. The prevalence of open-angle glaucoma among blacks and whites 73 years and older: the Salisbury Eye Evaluation Glaucoma Study. Arch Ophthalmol. 2006;124:1625-30.

36. Varma R, Tielsch JM, Quigley HA, Hilton SC, Katz J, Spaeth GL, et al. Race-, age-, gender-, and refractive errorrelated differences in the normal optic disc. Arch Ophthalmol. 1994;112:1068-76. 